\title{
Role of CT scan thorax in nCovid19-a case-based review
}

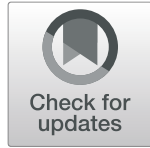

Vishnu sharma Moleyar ${ }^{1}$, Anupama Noojibail ${ }^{2 *}$, Nagaraj shetty $I^{3}$, Harsha D $S^{1}$ and Nithish Bhandary $\mathrm{M}^{3}$

\begin{abstract}
Background: Most of the morbidity and mortality in nCovid19 is due to pneumonia which can be reduced by early diagnosis and treatment. Chest CT scan plays an important role in the early diagnosis and management of respiratory complications due to $\mathrm{nCovid19}$. Clinicians should be aware about the indications for the CT scan of the thorax, timing of investigation, and limitations of $\mathrm{CT}$.

Main body of abstract: Chest CT scan is indicated in patients with moderate to severe respiratory symptoms and pretest probability of nCovid19 infection, when RT-PCR test results are negative, and in patients for whom an RT$P C R$ test is not performed or not readily available. When a rapid antigen test is negative and an RT-PCR test report takes time, CT can be used in seriously ill patients to decide whether it is COVID or not. For patients who are dependent on oxygen even after 2 weeks, CT may help to show the extent of lung involvement and predict longterm prognosis. CT may be done to exclude nCovid19 pneumonia. For patients with high risk for nCovid19 who require an immediate diagnosis to rule out lung involvement, $C T$ can be done. A normal $C T$ excludes nCovid19 pneumonia. CT scan is required in confirmed cases of nCovid19 pneumonia when complications are suspected clinically. These include pulmonary thromboembolism, pneumothorax, mediastinal/surgical emphysema, bacterial pneumonia, and unexplained deterioration with new shadows in chest X-ray. CT pulmonary angiogram is indicated when pulmonary embolism is suspected, and in other cases, plain CT should be done. In pre-operative cases where emergency surgery is required, nCovid19 disease is suspected clinically, and RT-PCR report awaited or not available, CT thorax can be done.

Conclusion: CT scan is useful for early diagnosis of lung involvement, detection complications, triaging of cases, risk stratification, and preoperative evaluation in select cases. CT scan should be done only when there is a definite indication so to reduce radiation hazards and to reduce health care expenditure. Normal CT excludes nCovid19 lung involvement, but the patient may have upper respiratory involvement which may progress later to involve lungs.
\end{abstract}

Keywords: Role of CT scan thorax, nCovid19, Viral bronchopneumonia

\section{Background}

nCovid19 disease starts as an upper respiratory illness. Some of these patients develop viral pneumonia. Most of the morbidity and mortality in nCovid19 is due to pneumonia [1]. Hence, early diagnosis and treatment of pneumonia due to nCovid19 is essential to reduce

\footnotetext{
* Correspondence: anupama.n@manipal.edu

${ }^{2}$ Department of Physiology, Kasturba Medical College, Mangalore, Manipal

Academy of Higher Education, Manipal, Karnataka, India

Full list of author information is available at the end of the article
}

morbidity and mortality. CT scan of the chest plays an important role in the early diagnosis and management of respiratory complications due to nCovid19 [2]. Clinicians should be aware about the indications for CT scan of the thorax, timing of the investigation, and limitations of CT. Unnecessary CT scans will add to healthcare costs and exposure to radiation.

Duration of onset of the first symptom of the disease in nCovid19 is very important. During the first 5 days, usually, the disease is confined to the upper respiratory 
tract. After the 5th day, usually lower respiratory involvement starts. Any time between 5 and 14 days, lower respiratory involvement can occur, though the maximum chance is between the 5th and 12th day [2]. After 14 days, thromboembolic complications can occur up to 3 to 6 months. Derangement in total white cell count, neutrophil/lymphocyte ratio, C-reactive protein, liver function tests, renal function tests, lactate dehydrogenase, D-dimer, and Ferritin may give some clue regarding the risk of complications [2].

The most important first step in the management of nCovid19 patients is a risk stratification for developing complications. For age less than 50 years, the absence of comorbidities, mild disease after 7 to 10 days, no underlying immunosuppression, and mild disease in a highrisk patient after 14 days of initial symptoms are usually at low risk to develop complications [3]. For the age more than 60 years, any one or more comorbidities like diabetes mellitus, obesity, systemic hypertension, ischemic heart disease, other cardiac illness, chronic renal disease, chronic liver disease, immunosuppression, underlying malignancy, any chronic organ disease, and anyone with moderate or severe disease are at risk for nCovid19-related complications [3].

The most common complication and cause of death in nCovid19 is viral bronchopneumonia [2]. Early diagnosis and treatment improve the outcome in these patients. Systemic steroids, anticoagulants, antiviral medications, and correction of hypoxia are essential in all patients with bronchopneumonia [3]. CT scan thorax is useful in detecting lung lesions early in these patients. In this case-based essay, we discuss the role of CT scan thorax in patients with or suspected nCovid19.

\section{Main body \\ Case 1}

A 42-year-old man confirmed with nCovid 19 by RTPCR with no comorbidities, day 7 after the onset of initial symptoms, was admitted. He has no cough, dyspnea, or fever. His vital signs are normal, $\mathrm{SpO}_{2}$ is $98 \%$ in room air, and chest X-ray is normal. Inflammatory markers were not raised. Is chest $\mathrm{CT}$ required now in this patient?

\section{Answer}

No. He has only mild disease. He has no risk factors to develop any complications. Hence, he just needs observation for up to 15 days for any complications.

\section{Learning point}

Chest imaging is not indicated in asymptomatic patients or in patients with mild respiratory symptoms of nCOVID-19 with a low risk to develop complications [4].
After the initial evaluation, this patient was discharged as the disease was mild. He was monitored for 15 days with home quarantine. He made an uneventful complete recovery.

\section{Case 2}

An 18-year-old female was admitted with fever, diarrhea, and lower abdominal pain since 3 days. She was diagnosed to have acute appendicitis by classical clinical signs and ultrasound abdomen. She required emergency surgery under general anesthesia. She had no respiratory symptoms. Vitals were normal. Respiratory system examination was normal, $\mathrm{SpO}_{2}-98 \%$ while breathing room air, no comorbidities, and no history of exposure or contact to nCovid19. Chest X-ray was normal. Creactive protein was raised. Other inflammatory markers were normal. The rapid antigen test was negative. RTPCR report will take 2 days as the sample load was heavy.

\section{Question}

Is CT scan thorax required before surgery?

\section{Answer}

Yes. Some patients with nCovid19 may present with GI symptoms and may not have any respiratory symptoms. CT may show early lung involvement if any, within 3-4 days. If CT is normal, the risk for postoperative complications will be less. If RT-PCR is readily available before surgery and is negative, a CT scan may not be essential in these types of cases.

\section{Learning point}

CT scan thorax may be done in pre-operative cases where emergency surgery is required, nCovid19 is suspected, or confirmatory report takes time [4]. This will help in risk stratification. The same applies to confirmed nCovid19 cases requiring emergency surgery since a CT scan will help find out early lung lesions if any.

CT thorax was normal in this patient. She underwent appendectomy and recovery without any complications.

\section{Case 3}

A 67-year-old man, confirmed with nCovid19, has diabetes mellitus, systemic hypertension, and ischemic heart disease and has no history of any lung diseases, day 6 from initial symptoms. He has a cough and progressive dyspnea for the last 2 days, $\mathrm{SpO}_{2}$ is $88 \%$ on room air, and cardiac and renal functions are normal. Has a 3-fold rise in D-dimer and a 4-fold rise in serum ferritin. Chest X-ray shows bilateral lower zone peripheral opacities (Fig. 1). 


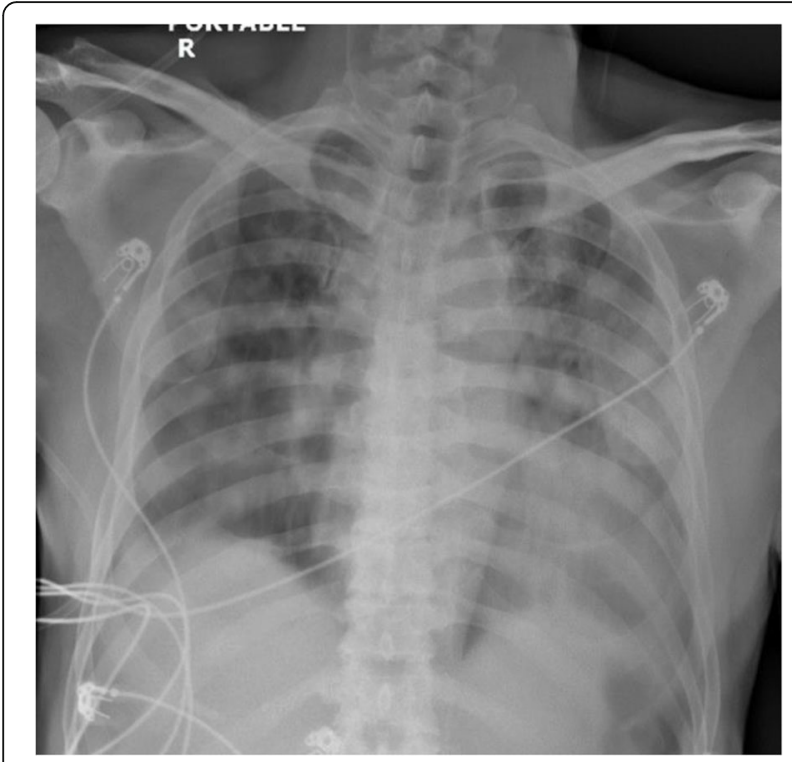

Fig. 1 Chest $X$-ray showing bilateral lower zone peripheral opacities

\section{Question}

Is a CT scan required in this patient?

\section{Answer}

No. Diagnosis of nCovid19 pneumonia is already confirmed by symptoms and chest X-ray. No other comorbid or premorbid lung disease is suspected.

\section{Learning point}

CT scan is not required if no additional pathology is suspected in confirmed nCovid19 bronchopneumonia where chest X-ray shows the lesions [4].

\section{Case 4 continued}

He progressively deteriorates despite the standard treatment. He was in the Intensive Care Unit for 15 days and has persistently high inflammatory markers and Ddimer. He is dependent on oxygen 10 li by a high concentration mask. No other contributing factors for hypoxia.

\section{Question}

Is chest $\mathrm{CT}$ required now?

\section{Answer}

Yes. CT thorax with CT pulmonary angiogram is needed to see for the extent of the parenchymal lesion and pulmonary embolism if any as patient has persistent hypoxia. Most of the patients recover from hypoxia within 15 days. Persistent hypoxia beyond 15 days needs evaluation.

\section{Learning point}

Chest CT is valuable to evaluate patients with clinical deterioration for nCOVID-19 progression or secondary cardiopulmonary complications such as acute respiratory distress syndrome, pulmonary embolism, superimposed pneumonia, or heart failure [4]. Contrast CT is required if pulmonary embolism or other conditions which require contrast is suspected. CT is also helpful in predicting long-term prognosis and outcome. Fibrosis and honeycombing indicate permanent changes and may indicate the need for long-term oxygen therapy in patients with persistent hypoxia.

There was no evidence of pulmonary embolism in this patient. CT showed extensive disease with honeycombing (Figs. 2 and 3). He made a slow recovery and was required with long-term home oxygen for 3 months.

\section{Case 5}

A 55-year-old male, obese, and diabetic patient was confirmed with nCovid19 pneumonia, day 9 after initial symptoms. He was admitted to the hospital for 2 days due to uncontrolled diabetes mellitus with raised markers and developed sudden worsening of breathlessness with hypoxia and tachycardia on day 9. No obvious cause for sudden worsening. Electrocardiogram, echocardiography, chest X-ray, and renal and liver function were normal.

\section{Question}

Does chest CT has any role in this patient?

\section{Answer}

Yes. CT scan thorax with CT pulmonary angiogram is indicated as sudden worsening with no obvious cause may be due to pulmonary embolism. CT will also show extent of parenchymal lesion if any.

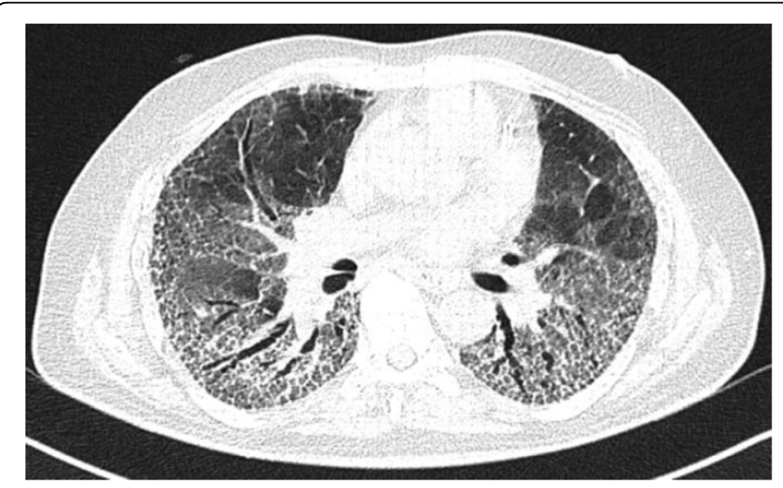

Fig. $2 C T$ scan lung window showing honeycombing with traction broncheictasis 


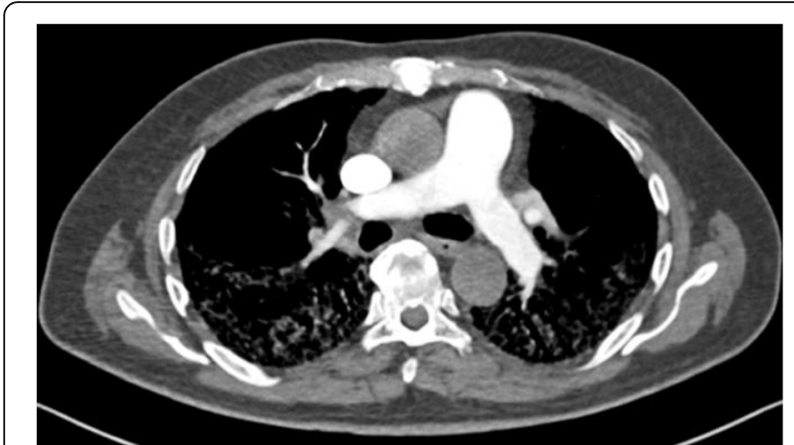

Fig. $3 \mathrm{CT}$ pulmonary angiogram - No evidence of pulmonary embolism

\section{Learning point}

CT may be required in unexplained worsening of symptoms. CT scan can detect small pneumothorax, pulmonary embolism, extent of lung involvement, and associated cardiopulmonary complications if any [4].

CT pulmonary angiogram showed bilateral small peripheral pulmonary emboli (Figs. 4 and 5). Despite all treatment, he progressively worsened requiring invasive ventilation. He finally succumbed on the 18th day.

\section{Case 6}

A 64-year-old male with anasarca, long standing diabetic with left foot infected gangrene, post-operative day 4 with fever, and cough was admitted. His $\mathrm{SpO}_{2}$ was $88 \%$ in room air. Chest X-ray showed right lower lobe infiltrates. No other obvious cause for respiratory symptoms. Rapid antigen test and RT-PCR for nCovid19 were negative. Cardiac evaluation was normal.

\section{Question}

Is there a role for $\mathrm{CT}$ in this patient?

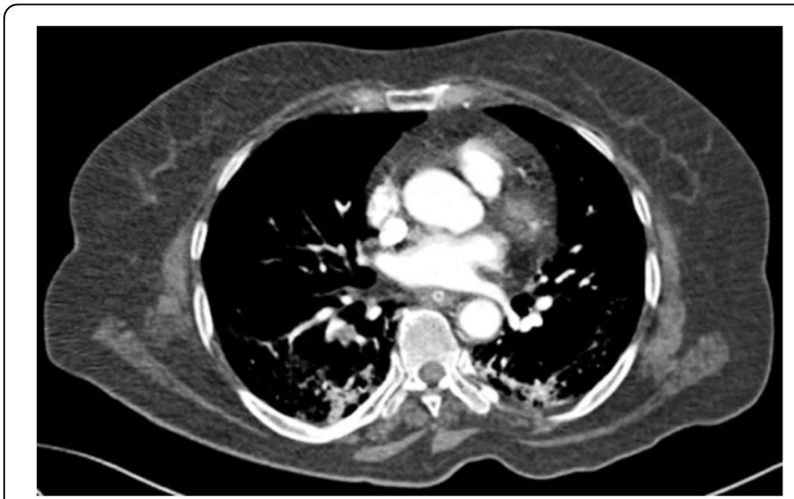

Fig. $4 C T$ pulmonary angiogram showing bilateral small peripheral pulmonary emboli

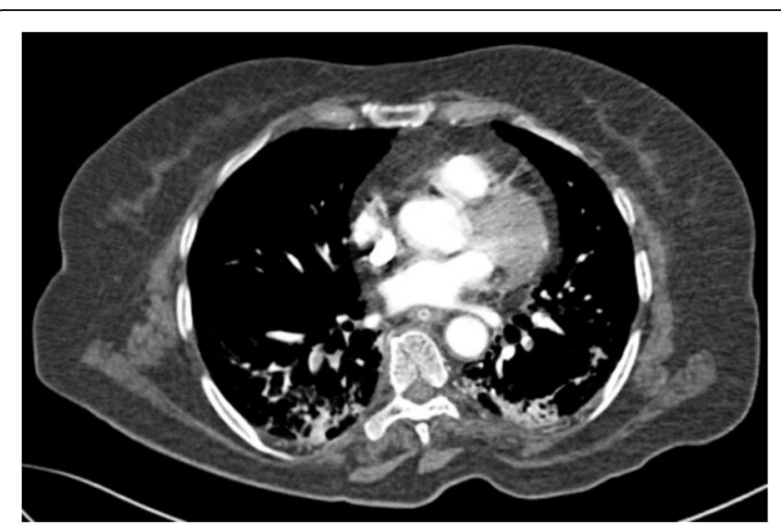

Fig. 5 CT pulmonary angiogram showing bilateral small peripheral pulmonary emboli

\section{Answer}

Yes. High-resolution CT will show parenchymal lesions if any. CT will help to determine the cause for lung infiltrates.

\section{Learning points}

CT scan of the chest is helpful to detect alternative diagnosis and exclude nCovid19 pneumonia [4]. Normal chest CT excludes nCovid19 pneumonia. CT is helpful to differentiate cardiogenic pulmonary edema, volume overload pulmonary oedema, and other conditions which can mimic nCovid19.

CT scan showed bilateral small pleural effusion in this patient. No parenchymal lesions suggestive of nCovid19 were visualized in the CT scan (Fig. 6). He made uneventful recovery with correction of hypoprotinemia and nutritional status.

\section{Case 7}

A 62-year-old male was admitted 9 days after the initial symptoms with increasing cough and dyspnea, since last 2 days. He has no comorbidities. $\mathrm{SpO}_{2}$ was $90 \%$ in room air. Chest X-ray showed bilateral lower lobe opacities. Rapid antigen test and RT-PCR were negative. All inflammatory markers are raised.

\section{Question}

Does CT has any roles?

\section{Answer}

Yes. CT will show parenchymal lesions. This will help to determine the probable cause for lung infiltrates.

\section{Learning point}

Chest imaging is indicated in patients with moderate to severe respiratory symptoms and any pre-test probability of nCovid19 infection, when RT-PCR test results are 


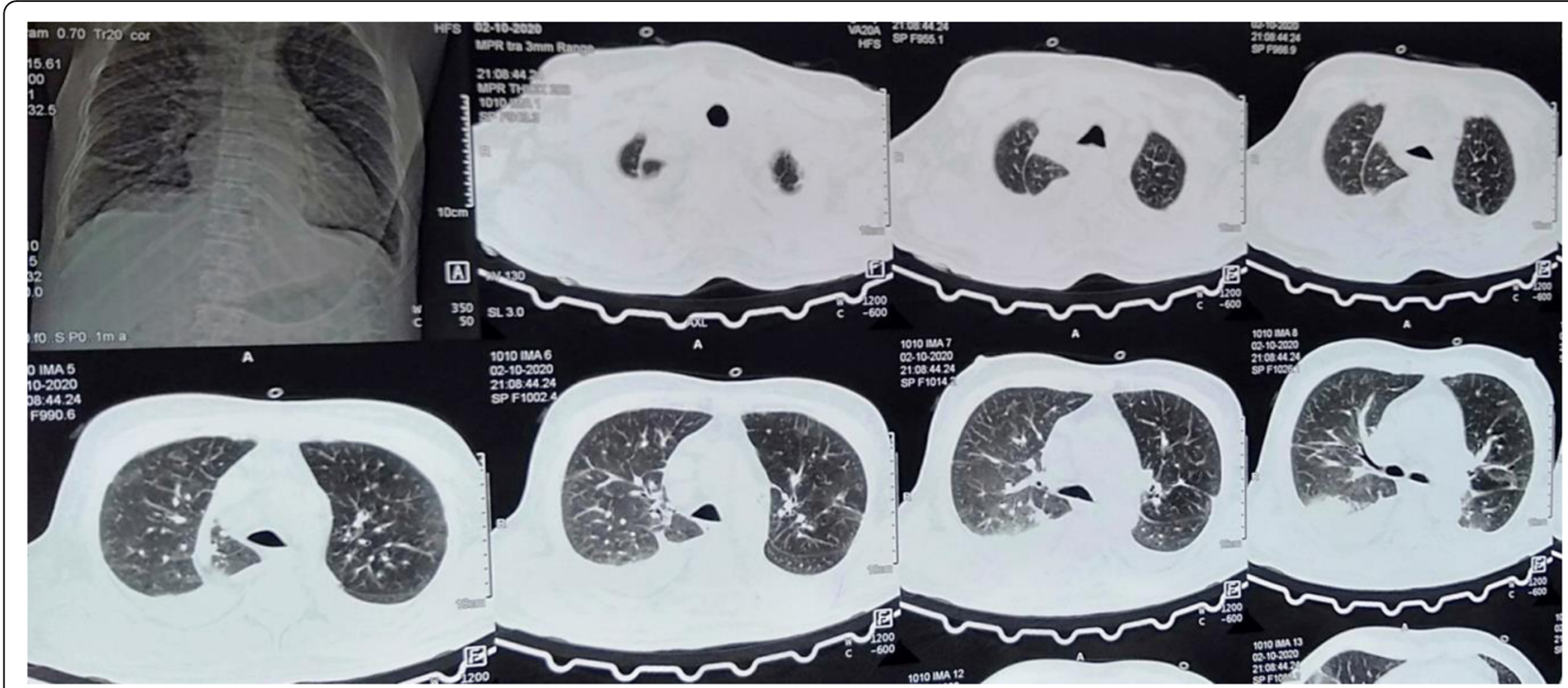

Fig. 6 CT scan showing bilateral small pleural effusion in this patient. No parenchymal lesions suggestive of nCovid19 were visualized in the CT scan

negative, and in any patient for whom an RT-PCR test is not performed or not readily available [4].

CT scan showed typical features of nCovid19 bronchopneumonia (Fig. 7), and this patient was diagnosed as RT-PCR-negative nCovid19 bronchopneumonia. He made an uneventful recovery with treatment. RT-PCR may be negative in up to $20 \%$ of patients with nCovid19 pneumonia. These cases are diagnosed by symptoms, CT scan, and exclusion of other causes [5].

\section{Case 8}

A 55-year-old male was diagnosed with nCovid19, day 14 after initial symptoms. He had a severe dry cough for 1 week and was admitted with facial swelling. On

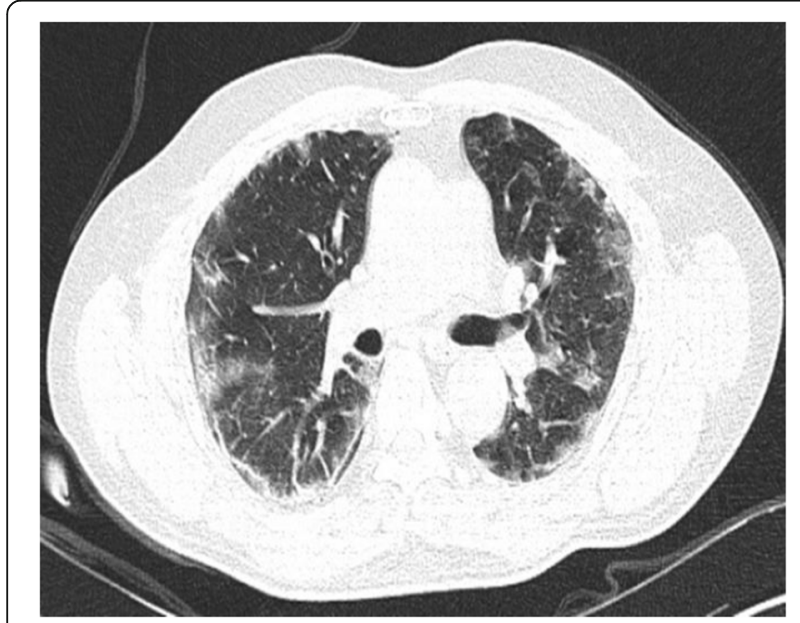

Fig. 7 CT scan showing typical features of nCovid19 bronchopneumonia examination, he has extensive surgical emphysema. Chest X-ray showed evidence of mediastinal and surgical emphysema, but did not show any evidence of pneumothorax (Fig. 8).

\section{Question}

Is $\mathrm{CT}$ required?

\section{Answer}

Yes. CT will be helpful to determine the cause for surgical emphysema, small pneumothorax if any which may be missed in chest X-ray, and extent of lung lesion. CT

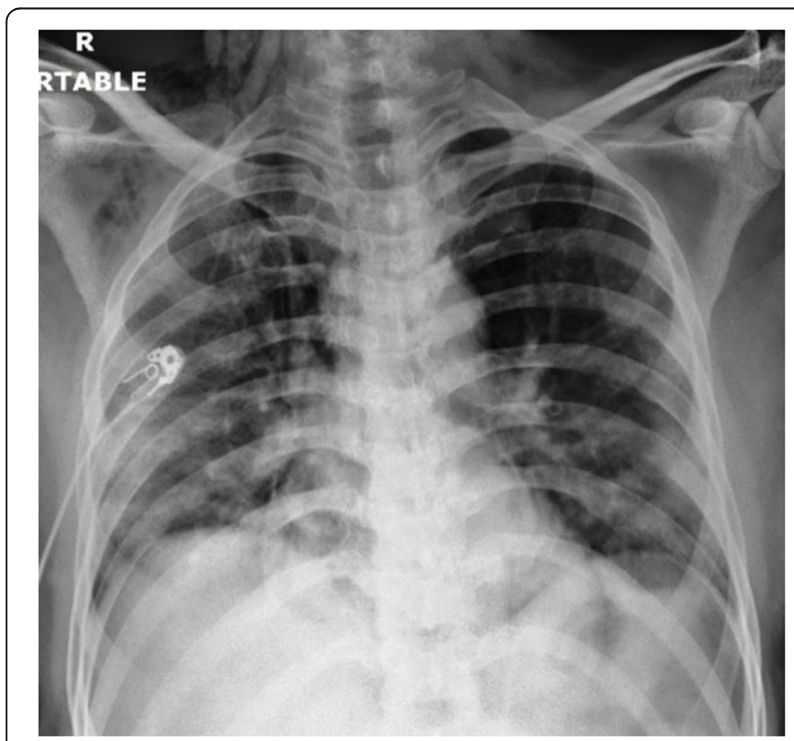

Fig. 8 Chest X-ray showing evidence of mediastinal and surgical emphysema, no evidence of pneumothorax 
is required if any complications are suspected during the course of the disease. In case of pneumothorax, an intercostal tube (ICT) is mandatory. If only surgical emphysema, ICT has no role.

\section{Learning point}

CT is required when complications/additional unexplained symptoms or X-ray lesions appear [4].

CT scan showed small right-sided pneumothorax, mediastinal, and surgical emphysema with typical parenchymal lesions suggestive of nCovid119 bronchopneumonia (Figs. 9 and 10). He eventually made an uneventful recovery with treatment.

\section{Case 9}

A 62-year-old man had a mitral valve replacement 3 years back; confirmed with nCovid19 disease, day 3 after initial symptoms; and has a cough and dyspnea for 1 day. $\mathrm{SpPO}_{2}$ was $91 \%$ in room air. Chest X-ray has no lung lesions (Fig. 11). The inflammatory markers were marginally raised, the cardiac evaluation did not reveal any new abnormality, the left ventricular ejection fraction was normal, and the mitral valve functions normally.

\section{Question}

Is there any role for chest $\mathrm{CT}$ ?

\section{Answer}

Yes. He is at high risk to develop complications and has early symptoms of dyspnea. Early diagnosis of pneumonia and aggressive treatment will improve the outcome.

\section{Learning point}

In high-risk symptomatic cases, early CT may be required. CT may be useful in high-risk cases for early diagnosis of nCovid19 pneumonia, usually $3-4$ days after the initial onset of symptoms [4].

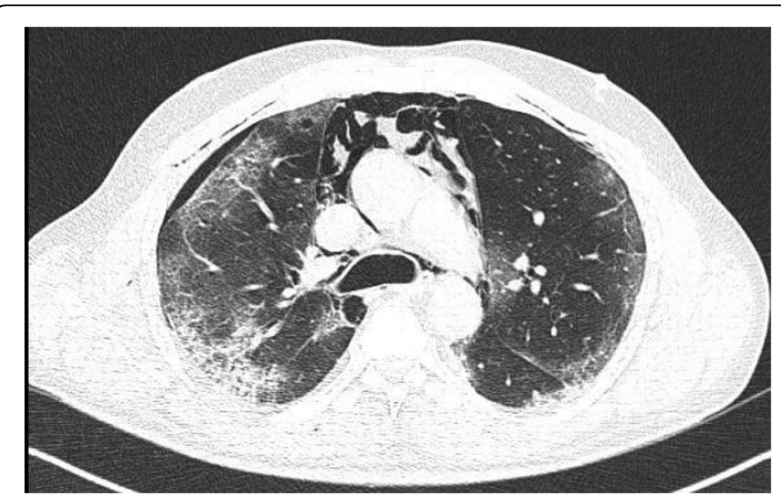

Fig. 9 CT scan thorax lung window showing small right sided pneumothorax, pneumomediastinum, surgical emphysema and lung parenchymal lesions typical of nCovid19 bronchopneumonia

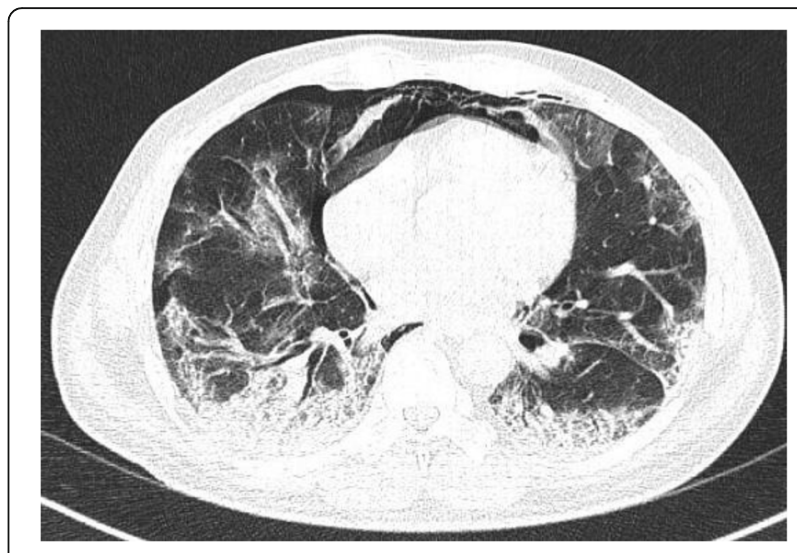

Fig. $10 C T$ scan thorax lung window showing small right sided pneumothorax, pneumomediastinum, surgical emphysema and lung parenchymal lesions typical of nCovid19 bronchopneumonia

Chest CT has been suggested to have potential value as a rapid triaging tool in patients with moderate to severe respiratory symptoms in a resource-constrained environment where nCovid-19 is highly prevalent [4].

His chest CT showed early ground-glass opacities (Figs. 12 and 13). He was initiated on early aggressive treatment, and he made an uneventful recovery.

\section{CT for triaging of cases}

When rapid antigen test is negative and RT-PCR test report takes time, CT can be used in sick, seriously ill symptomatic patients to differentiate between nCovid19 or non-Covid lung disease. Also, in case the patient requires emergency surgery and symptoms are equivocal, CT can be done. CT may be done to determine the duration of disease when history not clear as the duration from the onset of the disease is important for treatment [4].

Patients who are dependent on oxygen even after 2 weeks of CT may help to show the extent of lung involvement and predict long-term prognosis. CT may be repeated if there is unexplained worsening of hypoxia for any small pneumothorax, pulmonary embolism, or other conditions which can worsen symptoms.

\section{Case 10}

A 65-year-old patient was admitted for coronary artery bypass graft (CABG). This patient had no respiratory symptoms. He was treated as mild nCovid19 4 months back. Clinical examination of the respiratory system was normal on current admission. Resting $\mathrm{SpO}_{2}$ and 6-min walk test was normal. His chest CT showed few bilateral ground-glass opacities (Figs. 14, 15, and 16). Inflammatory markers were normal. 


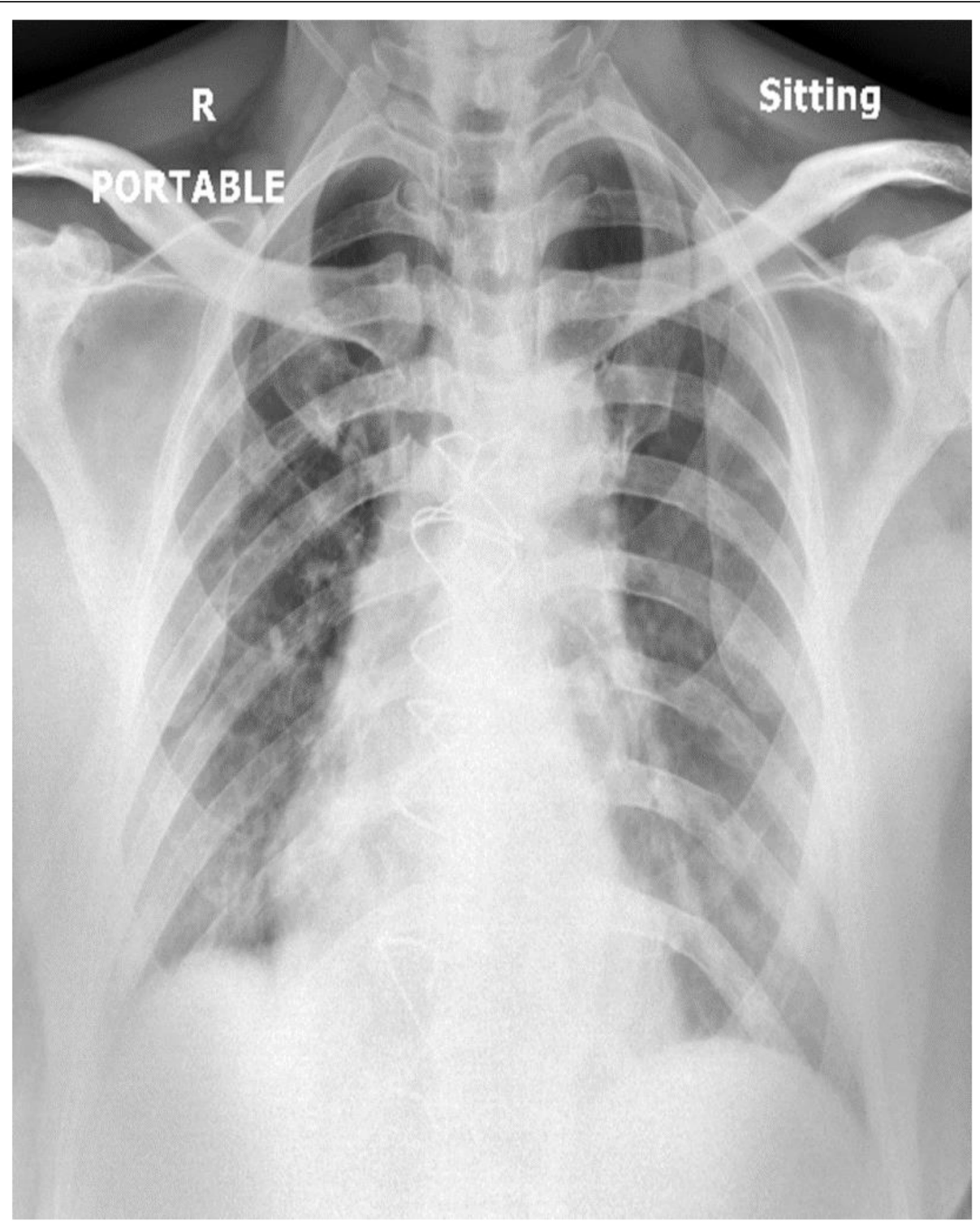

Fig. 11 Chest $X$-ray no lung lesions

\section{Question}

Is the lung lesion needs to be treated and does the patient require any postponement of surgery?

\section{Answer}

Since the patient is asymptomatic and investigations are normal, lung lesion does not need any treatment. Post nCovid19 parenchymal opacities may take time to heal, and residual scarring may persist. Since it is already 4 months after the infection, surgery can be done.

\section{Learning point}

Lung lesions may take time to heal, and residual scarring may occur in some patients. If these patients are asymptomatic, markers are normal, 6-min walk test is normal (no hypoxia on exertion), and no further treatment or evaluation is required [4]. Since it is already 4 months after the infection, surgery can be done.

He underwent CABG. He had no complications after CABG.

\section{Imaging modality}

Till recently, a high-resolution CT scan was preferred. Recent studies showed that low-dose CT protocol is a reliable diagnostic tool to detect nCovid-19 pneumonia. Low-dose chest CT protocol results in a remarkable reduction (up to 89\%) in the radiation dose compared to the standard-dose protocol without lowering diagnostic accuracy [6]. High-resolution CT is indicated only if any other diffuse parenchymal lung diseases are suspected. 


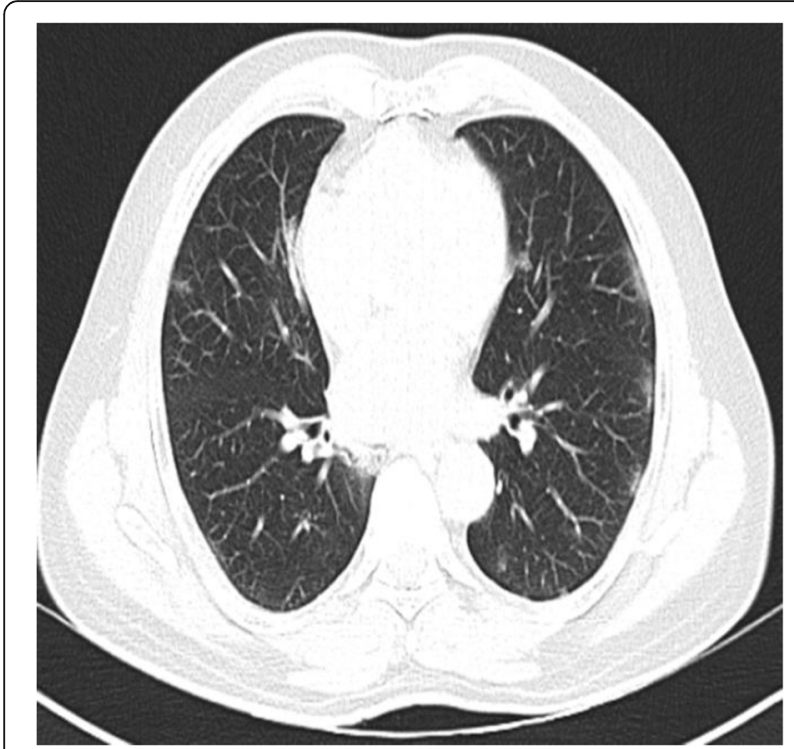

Fig. 12 Chest CT showing early ground glass opacities

Contrast CT is required if any other additional pathologies requiring contrast are suspected. CT pulmonary angiogram is required if pulmonary embolism is suspected.

\section{Summary}

Indications for CT scan thorax in nCovid19 For diagnosis

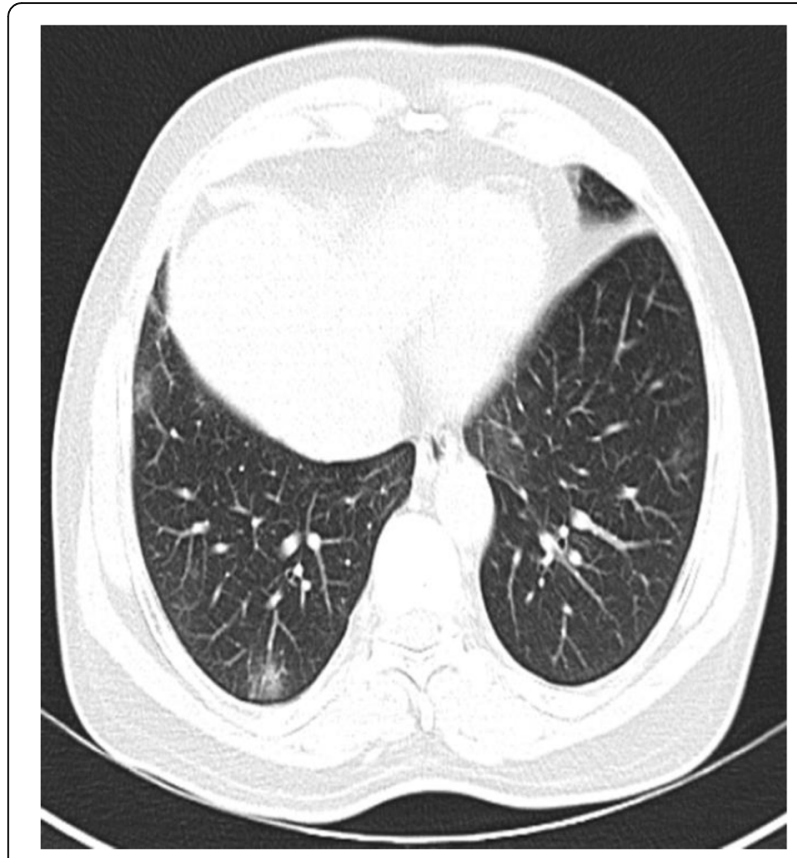

Fig. 13 Chest $C T$ showing early ground glass opacities

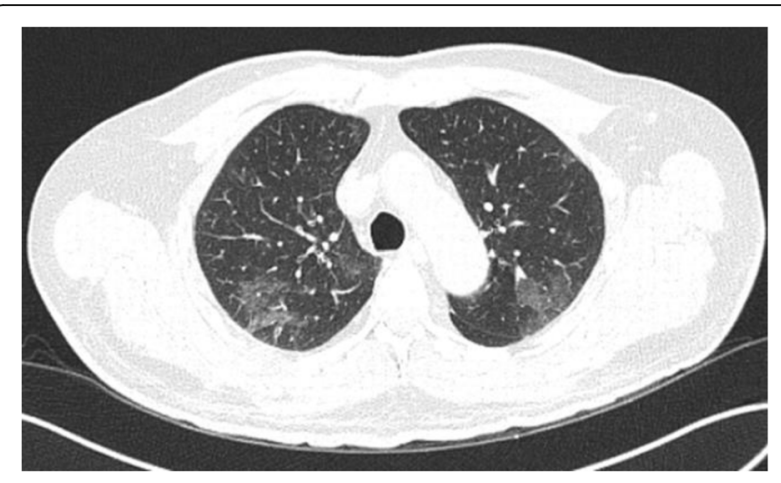

Fig. 14 Chest CT showing few bilateral ground glass opacities

1. Chest CT scan is indicated in patients with moderate to severe respiratory symptoms and pretest probability of nCovid19 infection, when RTPCR test results are negative, and in any patient for whom an RT-PCR test is not performed or not readily available. CT may be useful in high-risk cases for early diagnosis of nCovid19 pneumonia. In high-risk cases without any respiratory symptoms, CT should be done after the 4th day of onset of first symptoms as lung involvement usually occurs after the 4th day.

2. Chest CT scan has been suggested to have potential value as a rapid triaging tool in patients with moderate to severe respiratory symptoms in a resource-constrained environment where nCovid19 is highly prevalent. When a rapid antigen test is negative and an RT-PCR test report takes time, CT can be used in seriously ill patients to decide whether it is Covid or non-Covid.

3. Patients who are dependent on oxygen even after 2 weeks, CT may help to show the extent of lung involvement and predict long-term prognosis.

4. CT may be done to exclude nCovid19 pneumonia. In patients with high risk for nCovid19 who require

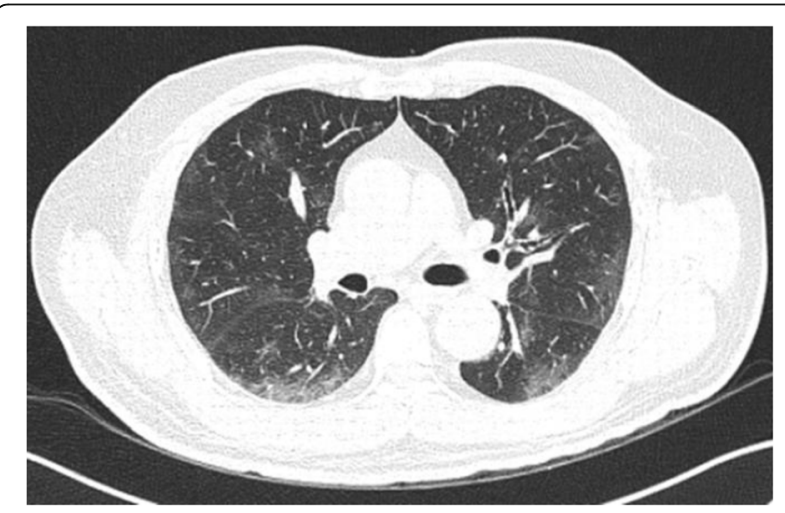

Fig. 15 Chest CT showing few bilateral ground glass opacities 


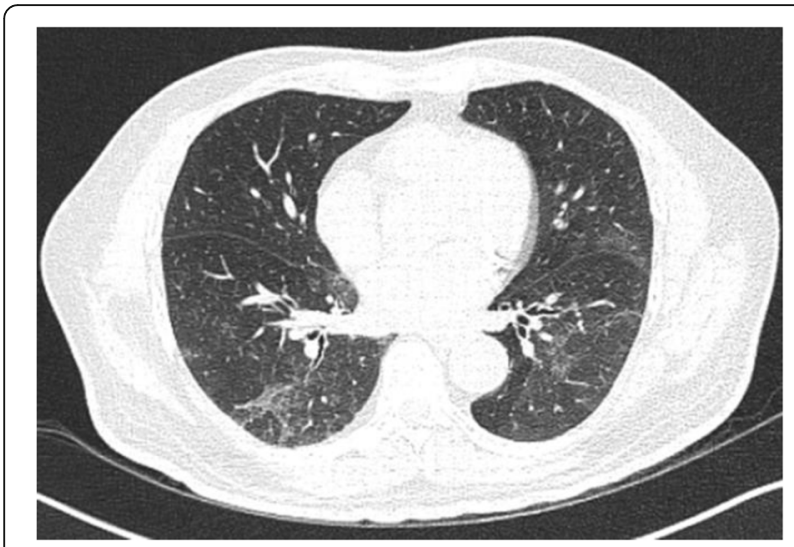

Fig. 16 Chest $C T$ showing few bilateral ground glass opacities

an immediate diagnosis to rule out lung involvement, CT may be done. A normal CT excludes nCovid19 pneumonia. But we should remember a normal chest CT does not exclude nCovid19 infection, and the patient may still have upper respiratory disease. CT can also suggest an alternative diagnosis like pulmonary edema as a cause for symptoms.

\section{To detect complications}

1. CT scan may be required in confirmed cases of nCovid19 pneumonia when complications are suspected clinically. These include pulmonary thromboembolism, pneumothorax, mediastinal/ surgical emphysema, bacterial pneumonia, and unexplained deterioration with new shadows in chest X-ray. CT pulmonary angiogram is indicated when pulmonary embolism is suspected. In all the other cases, plain CT should be done.

\section{Pre-operative}

This is for risk stratification and to identify nCovid19 pneumonia early. Pre-operative cases where emergency surgery is required, nCovid19 disease is suspected clinically, RT-PCR report awaited or not available CT thorax can be done.

\section{Where chest CT is not essential}

1. Chest CT scan is not indicated in asymptomatic patients or in patients with mild respiratory symptoms of nCovid19 with low risk. Anyone who is less than 50 years of age, without any comorbidities, no immunosuppression, and mild disease in a high-risk patient after 14 days of initial symptoms are classified as low-risk cases.
2. In a confirmed case where a chest X-ray shows pneumonia, CT thorax is not essential if no complications are suspected.

3. Follow-up CT scan is not required if the patient is improving clinically and no complications are suspected.

4. Lung involvement after 14 days of onset of first symptoms is unusual. Hence, a CT of the chest is not required even in high-risk cases in the absence of any symptoms or signs of pneumonia after the 14th day of onset of first symptoms.

\section{Limitations of CT}

Availability, expertise for interpretation, cost, and radiation, non-portable, that may not be feasible to repeat are the limitations. CT is not the standard for the diagnosis of nCovid19, but its findings help suggest the diagnosis in the appropriate setting.

Chest CT findings should be correlated with epidemiologic history, clinical presentation, and RT-PCR test results. Many other diseases can mimic nCovid19 pneumonia in CT and vice versa [7]. Typical or indeterminate features of nCovid19 pneumonia may be incidentally detected at CT performed for other reasons. In these cases, the interpreting radiologist should discuss the possibility of nCovid19 with the referring physician in a timely manner. Chest CT should not be used as an independent diagnostic tool to exclude or confirm nCovid19. Chest CT can be done in stroke patients and myocardial infarction if nCovid19 is suspected as the predisposing event. The possibility of nCovid19 related cardiac injury should be kept as a differential diagnosis when the pericardial effusion is depicted on chest CT images. A normal chest CT examination result does not exclude nCovid19 infection but excludes nCovid19 pneumonia. The proportion of false-positive chest CT examination results is substantial. This is due to the overlapping imaging features with numerous other diseases, including other viral cases of pneumonia. The positive predictive value for chest CT is low (1.5 to $30.7 \%$ ) in low-prevalence regions, and the negative predictive value ranges from 95.4 to $99.8 \%$ for COVID pneumonia [8]. Pooled sensitivity and specificity for chest CT is 94 to $96 \%$ and $37 \%$, respectively.

\section{Conclusions}

CT scan is useful for early diagnosis of lung involvement, detection complications, triaging of cases, risk stratification, and preoperative evaluation in select cases. CT scan should be done only when there is a definite indication to reduce radiation hazards and to reduce health care expenditure.

Findings of CT scan chest should be correlated with history, physical findings, and comorbid diseases and 
consider other differentials. Normal CT excludes nCovid19 lung involvement but the patient may have upper respiratory involvement which may progress later to involve the lungs. Diagnosis of nCovid19 infection should be confirmed by RT-PCR.

\section{Abbreviations}

$\mathrm{CT}$ : Computed tomography; $\mathrm{SpO}_{2}$ : Saturation of peripheral oxygen

\section{Acknowledgements}

Dr. Ganesh K., Professor \&Head, Department of Radiodiagnosis, A J Institute of Medical Sciences \& Research Centre, Kuntikana, Mangalore.

\section{Declarations}

\section{Authors' contributions}

VSM---collection and interpretation of patient details, review of literature, and manuscript preparation

AN--- review of literature and manuscript preparation

INS---collection and interpretation of patient details and manuscript preparation

NBM---collection and interpretation of patient details and manuscript preparation

HDS---collection and interpretation of patient details and manuscript preparation

The authors have read and approved the final manuscript and ensure that this is the right case.

\section{Funding}

NIL

\section{Availability of data and materials}

Available in the A J Institute of Medical Sciences \& Research Centre,

Kuntikana, Mangalore

\section{Ethics approval and consent to participate}

Approved by the institutional ethical committee of A J Institute of Medical Sciences \& Research Centre, Kuntikana, Mangalore

\section{Consent for publication}

Consent from all the participants is taken.

\section{Competing interests}

The authors declare that they have no competing interests.

\section{Author details}

${ }^{1}$ Respiratory Medicine, A.J. Institute of Medical Sciences \& Research Centre, Kuntikana, Mangalore, Karnataka, India. ${ }^{2}$ Department of Physiology, Kasturba Medical College, Mangalore, Manipal Academy of Higher Education, Manipal, Karnataka, India. ${ }^{3}$ Internal Medicine, A.J. Institute of Medical Sciences \&

Research Centre, Kuntikana, Mangalore, Karnataka, India.

Received: 5 May 2021 Accepted: 7 June 2021

Published online: 18 June 2021

\section{References}

1. Chen N, Zhou M, Dong X, Qu J, Gong F, Han Y, Qiu Y, Wang J, Liu Y, Wei Y, Xia J', Yu T, Zhang X, Zhang L (2020) Epidemiological and clinical characteristics of 99 cases of 2019 novel coronavirus pneumonia in Wuhan, China: a descriptive study. Lancet. 395(10223):507-513. https://doi.org/10.1 016/S0140-6736(20)30211-7

2. Yang W, Cao Q, Qin L, Wang X, Cheng Z, Pan A, Dai J, Sun Q, Zhao F, Qu J, Yan F (2020) Clinical characteristics and imaging manifestations of the 2019 novel coronavirus disease (COVID-19): a multi-center study in Wenzhou city, Zhejiang, China. J Inf Secur 80(4):388-393. https://doi.org/10.1016/j.jinf.2020. 02.016

3. National Institute for Health and Care Excellence (2021) COVID-19 rapid guideline: managing COVID-19. internet publication
4. Rubin GD, Ryerson CJ, Haramati LB, Sverzellati N, Kanne JP, Raoof S et al (2020) The role of chest imaging in patient management during the COVID19 pandemic: a multinational consensus statement from the Fleischner Society. Radiology 296(1):172-180

5. Bhandary NM, Sharma V, Megha SN (2021) Rapidly progressive dyspnea in an elderly diabetic. J Adv Lung Health 1:31-34

6. Azadbakht J, Khoramian D, Lajevardi ZS, Elikaii F, Aflatoonian AH, Farhood B, Najafi M, Bagheri H (2021) A review on chest CT scanning parameters implemented in COVID-19 patients: bringing low-dose CT protocols into play. Egypt J Radiol Nucl Med 52(1):13. https://doi.org/10.1186/s43055-02000400-1

7. Kwee TC, Kwee RM (2020) Chest CT in COVID-19: what the radiologist needs to know. RSNA -Published Online. https://doi.org/10.1148/rg.2020200159

8. Hossein H, Ali KM, Hosseini M et al (2020) Value of chest computed tomography scan in diagnosis of COVID-19: a systematic review and metaanalysis. Clin Transl Imaging 12:1-13

\section{Publisher's Note}

Springer Nature remains neutral with regard to jurisdictional claims in published maps and institutional affiliations.

\section{Submit your manuscript to a SpringerOpen ${ }^{\circ}$ journal and benefit from:}

- Convenient online submission

- Rigorous peer review

- Open access: articles freely available online

- High visibility within the field

- Retaining the copyright to your article

Submit your next manuscript at $\boldsymbol{\nabla}$ springeropen.com 